\title{
Magnetically Induced Current Piston for Generating Extreme-ultraviolet Fronts in the Solar Corona
}

\author{
Pakorn Wongwaitayakornkul ${ }^{1,3}$ (1), Magnus A. Haw ${ }^{1,3}$ (1D, $\mathrm{Hui} \mathrm{Li}^{2}$ (1), and Paul M. Bellan ${ }^{1}$ (1) \\ ${ }^{1}$ Applied Physics, California Institute of Technology, Pasadena, CA 91125, USA; pwongwai@ caltech.edu \\ 2 Theoretical Division, Los Alamos National Laboratory, Mail Stop B227, Los Alamos, NM 87545, USA \\ Received 2018 October 30; revised 2019 February 4; accepted 2019 February 21; published 2019 April 1
}

\begin{abstract}
Single-pulse, globally propagating coronal fronts, called Extreme-ultraviolet (EUV) waves, were first observed in 1995 by the Extreme-ultraviolet Imaging Telescope and every observed EUV wave since has been associated with a coronal mass ejection (CME). The physical mechanism underlying these waves has been debated for two decades with wave or pseudo-wave theories being advocated. We propose a hybrid model where EUV waves are compressional fronts driven by a reverse electric current layer induced by the time-dependent CME core current. The reverse current layer flows in a direction opposite to the CME core current and is an eddy current layer necessary to maintain magnetic flux conservation above the layer. Repelled by the core current, the reverse current layer accelerates upward so it acts as a piston that drives a compressional perturbation in the coronal regions above. Given a sufficiently fast piston speed, the compressional perturbation becomes a shock that separates from the piston when the piston slows down. Since the model relates the motion of the EUV front to CME properties, the model provides a bound for the core current of an erupting CME. The model is supported and motivated by detailed results from both laboratory experiments and ideal 3D magnetohydrodynamic simulations. Overlaps and differences with other models and spacecraft observations are discussed.
\end{abstract}

Key words: magnetohydrodynamics (MHD) - methods: laboratory: atomic - methods: numerical - plasmas - Sun: coronal mass ejections (CMEs)

\section{Introduction}

Globally propagating coronal fronts were first observed in 1995 by the Extreme-ultraviolet Imaging Telescope (EIT) on the Solar and Heliospheric Observatory. These "wave"-like structures, now called EIT waves or EUV waves, exhibit bright, nearly circular fronts in the EUV spectrum, with velocities in the range 200-500 $\mathrm{km} \mathrm{s}^{-1}$ (Klassen et al. 2000; Thompson \& Myers 2009; Muhr et al. 2014). In this work, we will follow the convention of Cohen et al. (2009) and describe these features as EUV waves. Spectral observations across a large temperature range (1-4 MK) indicate that these fronts are compressive perturbations (Warmuth et al. 2005; Kozarev et al. 2011; Ma et al. 2011). Additionally, there is evidence for adiabatic heating implying modest temperature increases of 5\%-10\% (WillsDavey \& Thompson 1999; Gopalswamy \& Thompson 2000; Schrijver et al. 2011; Vanninathan et al. 2015). To date, every observed EUV wave has been associated with a coronal mass ejection (CME; Biesecker et al. 2002; Warmuth et al. 2005; Veronig et al. 2008). Since failed eruptions and non-eruptive flares do not produce these fronts, the generation mechanism must be related to the CME expansion. EUV waves are also occasionally coincident with Type II radio bursts, indicating the presence of a shock (Carley et al. 2013).

Reviews of EUV waves (Wills-Davey \& Attrill 2009; Chen \& Fang 2011; Long et al. 2017) have divided the proposed theories into two groups: wave theories and pseudo-wave theories. A pseudo-wave is a phenomenon that behaves similarly to a wave, but is not prescribed by a wave equation. This separation characterizes a fundamental physical difference: waves are self-propagating and pseudo-waves require a driving mechanism. In the wave interpretation, EUV waves are

\footnotetext{
${ }^{3}$ These authors contributed equally to this work.
}

commonly thought to be fast-mode Alfvén waves/shocks (Thompson et al. 1998; Vršnak \& Cliver 2008) or some form of slow-mode soliton (Wills-Davey et al. 2007). The three pseudo-wave theories propose that the bright front is either (i) a current shell (Delannée et al. 2008), (ii) the wake of a Moreton wave (Chen et al. 2002), or (iii) reconnection at the expanding CME surface (Attrill et al. 2007). The different theories are categorized in Figure 1, based on the feature speed and timescale of the driver.

Unfortunately, none of the wave or pseudo-wave theories are consistent with all of the observed properties of EUV waves (Wills-Davey et al. 2007; Long et al. 2017). A suitable theory must explain why EUV waves (1) are observed as single pulses, (2) propagate at sub-Alfvénic speeds, (3) have coherence across solar diameter scales, and (4) can produce shocks. Due to these varied properties, many papers (Cohen et al. 2009; Liu et al. 2010; Downs et al. 2011; Cheng et al. 2012; Olmedo et al. 2012) have called into question the stark separation between the wave and pseudo-wave theories, instead calling for a hybrid model where both pseudo-wave and wave components are present.

This work is an extension of the cavity model in Haw et al. (2018) with an emphasis on the piston-driven shock mechanism. We describe in Section 2 a simple analytic model for an expanding compressional current layer at the surface of a CME and show that this model is consistent with all the observed properties of EUV waves. This hybrid model quantifies the magnetic driving mechanism, the dynamical evolution of the compressional front, and the generation of fast-mode waves/ shocks. The model is supported and motivated by an experiment of an erupting flux rope and by a 3D magnetohydrodynamic (MHD) simulation of this experiment, described in Sections 3 and 4, respectively. The experiment shows a visible $H_{\alpha}$ front with an associated reverse current layer propagating ahead of the main current channel. The simulation 


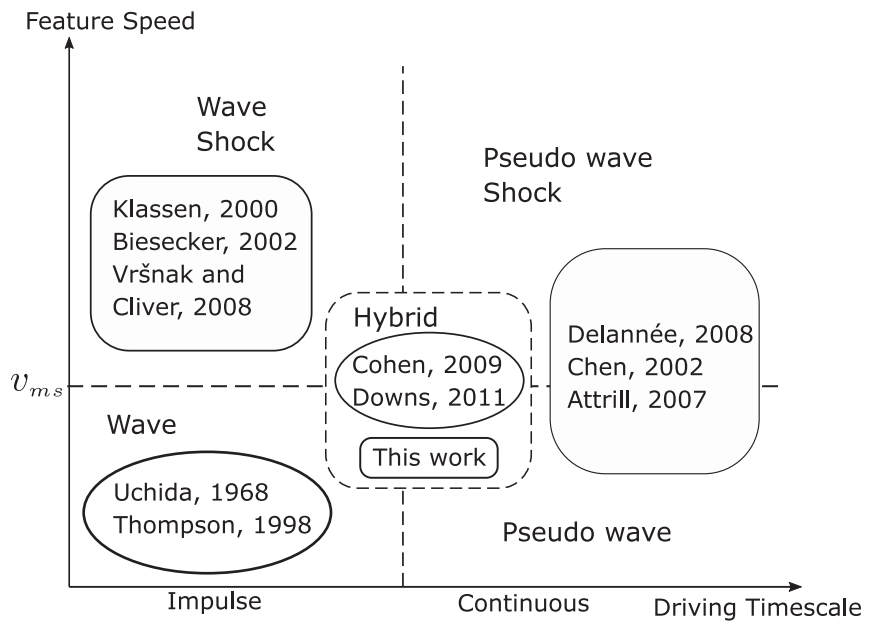

Figure 1. Classification of previous literature by feature speed and driving timescale. The trend of the proposed theories goes from wave, to shock, then to pseudo-wave, and finally to hybrid model. $v_{\mathrm{ms}}$ represents the local wave speed.

shows the generation of a propagating compressional layer that can be simultaneously classified as a fast-mode shock, a current shell, and the expanding surface of the CME. Section 5 discusses the degree to which the competing EUV wave theories are physically equivalent.

\section{Theory}

\subsection{Piston-driven Shock}

A shock is a discontinuity in plasma parameters that occurs when the plasma flow velocity exceeds the characteristic wave speed. In the frame moving with the shock, the plasma conserves mass flux, momentum, magnetic flux, and energy, while entropy increases across the shock. Equating the conserved quantities across the shock yields the RankineHugoniot (RH) jump conditions. In MHD fluids, information can be carried via three possible waves: (i) shear Alfvén wave, and (ii) fast and (iii) slow branch of magnetosonic wave, which result in three different shock types according to the associated wave. The fast magnetosonic shock is the only possible type for a configuration with a magnetic field perpendicular to the shock normal. In this case, the characteristic velocity of a perpendicular fast magnetosonic wave is $v_{\mathrm{ms}}=\sqrt{c_{s}^{2}+v_{\mathrm{A}}^{2}}$, where $c_{s}=\sqrt{\gamma P / \rho}$ and $v_{\mathrm{A}}=B / \sqrt{\mu_{0} \rho}$ are respectively the local sound and Alfvén speeds. Consider a 1D planar shock and suppose a tangential magnetic field $B_{t}$ is perpendicular to the normal flow velocity $v_{n}$; the following four quantities are conserved across the shock so their upstream $(u)$ and downstream $(d)$ values in the shock frame are equal: (i) mass, (ii) flux, (iii) momentum, and (iv) energy. Using $[Q]_{u}^{d}$ to denote the difference of a quantity $Q$ at upstream and downstream locations, the conservation equations can be written as

$$
\begin{gathered}
{\left[\rho v_{n}\right]_{u}^{d}=0,} \\
{\left[B_{t} v_{n}\right]_{u}^{d}=0,} \\
{\left[\rho v_{n}^{2}+p+\frac{B_{t}^{2}}{2 \mu_{0}}\right]_{u}^{d}=0,}
\end{gathered}
$$

$$
\left[\frac{1}{2} \rho v_{n}^{3}+\frac{\gamma}{\gamma-1} p v_{n}+v_{n} \frac{B_{t}^{2}}{\mu_{0}}\right]_{u}^{d}=0 .
$$

Solving the system of equations above (Fitzpatrick 2014) gives the relations

$$
\begin{gathered}
\frac{\rho_{d}}{\rho_{u}}=\frac{v_{n, u}}{v_{n, d}}=\frac{B_{t, d}}{B_{t, u}}=X, \\
\frac{p_{d}}{p_{u}}=Y=1+\gamma \mathcal{M}^{2}\left(1-X^{-1}\right) \\
+\beta^{-1}\left(1-X^{2}\right),
\end{gathered}
$$

where $X$ is the positive root of

$$
\begin{aligned}
& 2(2-\gamma) X^{2}+\left(2 \beta+(\gamma-1) \beta \mathcal{M}^{2}+2\right) \gamma X \\
& -\gamma(\gamma+1) \beta \mathcal{M}^{2}=0,
\end{aligned}
$$

$\mathcal{M}=v_{n, u} / \sqrt{\gamma p_{u} / \rho_{u}}$ is the upstream acoustic Mach number, and $\beta=2 \mu_{0} p_{u} / B_{t, u}^{2}$ is the ratio of the gas pressure to the magnetic pressure of the unshocked plasma. The expression for the compression ratio simplifies to $X=(\gamma+1) \mathcal{M}^{2} /$ $\left[2+(\gamma-1) \mathcal{M}^{2}\right]$ for a hydrodynamic shock $(\beta \gg 1, \mathcal{M})$ and $X=(\gamma+1) /(\gamma-1)$ for a strong shock $(\mathcal{M} \gg 1, \beta)$.

We can also show that the change of entropy, defined by $S=\ln \left(p / \rho^{\gamma}\right)$, can be expressed as $[S]_{u}^{d}=\ln Y-\gamma \ln X$. The second law of thermodynamics requires $[S]_{u}^{d}>0$. Since $d[S]_{u}^{d} / d X \geqslant 0$ everywhere and $[S]_{u}^{d}=0$ when $X=1$, it follows that $X>1$, i.e., the shock has to be compressive. Consequently, $B_{t, d}>B_{t, u}$ always, implying the existence of a current sheet.

The large amplitude wave driven by the piston steepens into a shock due to nonlinear evolution of the wavefront (Mann 1995; Vršnak \& Lulić 2000). The large amplitude wave continues to steepen until the width of the discontinuity reduces to either a dissipative or a dispersive length scale. The steepening and dissipative (or dispersive) effects balance out each other and the shock is formed. This implies that the shock will ultimately be formed in every wave with decreasing density in the direction of propagation (Landau \& Lifshitz 1959). The shock can form even when the piston moves below the characteristic wave speed (Žic et al. 2008), but it might not be observable due to the formation time being larger than the time of observation. When the piston travels at a speed above the characteristic wave speed, the shock is guaranteed to occur. When the piston decelerates, the shock retains its shape and propagation, separating from the piston.

\subsection{Return Current Layers as Magnetic Pistons}

This section outlines a simple analytic model for the generation and expansion of a compressional current layer at the surface of a CME. Consider a stable flux rope in the solar corona and suppose that, through some form of photospheric driving, the net current through this flux rope is increasing. This increasing current will also induce a thin anti-parallel current in the background plasma to shield the increasing azimuthal flux of the rising flux rope (Figure 2). This induced reverse current effectively creates a coaxial current distribution. Reverse currents are generated for all simulation boundarydriving mechanisms, including flux injection, shearing motion, and rotational motion (Tokman \& Bellan 2002; Lynch et al. 2004; Chen et al. 2006; Delannée et al. 2008). 


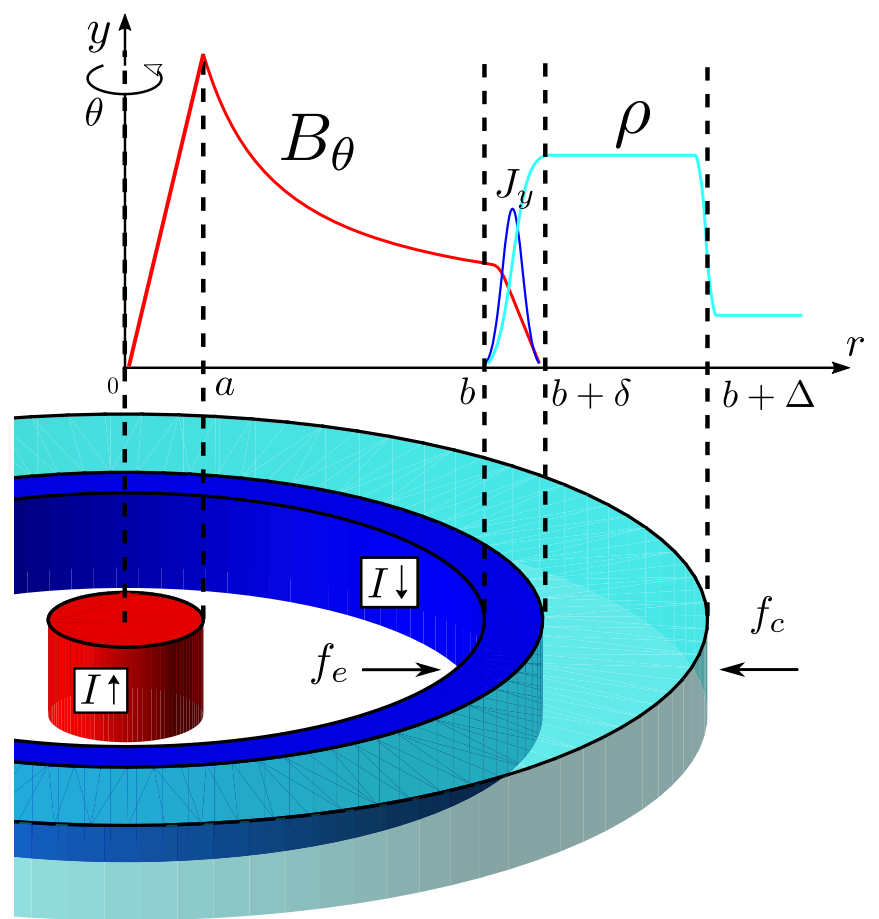

Figure 2. Illustration for the analytic model of the current layer generation and propagation.

Any coaxial current distribution tends to have separation between the forward and reverse currents because anti-parallel currents repel. Consequently, the return current layer will be forced away from the core current, forming a cavity region in between (Haw et al. 2018). The expanding reverse current layer effectively serves as a magnetic piston pushing background plasma out in the radial direction (Figure 2). If the expansion velocity of the current layer approaches the magnetosonic speed, the layer can develop a shock front. This mechanism was used in shock tube experiments in the 1960s to study highMach number shocks (Greifinger \& Cole 1961; Hoffman 1967) and is sometimes referred to as the inverse-pinch effect.

The inverse-pinch mechanism can be analytically modeled as a vertical $(\hat{y})$ increasing current $I(t)$ of radius $a$ in a uniform plasma of density $\rho_{0}$, magnetic field $B_{0} \hat{y}$, and pressure $P_{0}$. This rising current induces an anti-parallel shell current of width $\delta$, total current $-I$, and a constant current density $J_{y}=-I /[\pi(2 b+\delta) \delta]$ across its width. The central and return currents correspond to the respective red and dark blue features in Figure 2. The current shell (dark blue) pushes out plasma that is compressed into a density shell (light blue) of thickness $\Delta$, bounded by a shock front at $b+\Delta$.

The motion of the plasma density shell is controlled by two opposing forces: an expansive magnetic force $\left(f_{e}\right)$, which is proportional to the square of the central current $I$ and a confining force from background pressure $\left(f_{c}\right)$. Assuming an axisymmetric expansion of the current layer, we can express the 1D equation of motion in units of force-per-length as

$$
\frac{d}{d t}\left(M \frac{d}{d t} b(t)\right)=f_{e}-f_{c}
$$

where $M=\rho_{0} \pi\left((b(t)+\Delta)^{2}-a^{2}\right)$ is the total mass perlength compressed into the reverse current annulus, $b(t)$ is the inner radius of the reverse current annulus, $f_{c}$ is the confining force-per-length, and $f_{e}$ is the expansive force-per-length. The compression of all mass in the swept area into a dense exterior layer is called the "snowplow assumption" (i.e., $\delta, \Delta \rightarrow 0$ ) and represents the limiting behavior at high Mach number.

The total expansive force and confining force can be calculated as shown in Haw et al. (2018), giving the normalized integrable equation of motion for the magnetic piston to be

$$
\bar{M} \ddot{\bar{b}}=\frac{\bar{I}^{2}}{3 \pi} \frac{3 \bar{b}+2 \bar{\delta}}{(2 \bar{b}+\bar{\delta})^{2}}-\pi(\bar{b}+\bar{\Delta})[1+\beta]-\dot{\bar{M}} \dot{\bar{b}}
$$

where normalized values are indicated with a bar, i.e., $\bar{b}=b / a, \quad \bar{\delta}=\delta / a, \quad \bar{\Delta}=\Delta / a, \quad \bar{t}=\left(B_{0} / a \sqrt{\mu_{0} \rho_{0}}\right) t, \quad \bar{I}=$ $I / I_{0}=I \mu_{0} /\left(B_{0} a\right), \quad \bar{M}=M /\left(\rho_{0} a^{2}\right)=\pi\left((\bar{b}+\bar{\Delta})^{2}-1\right)$, and $\beta=2 \mu_{0} P_{0} / B_{0}^{2}$. This choice of normalization has three free parameters: background magnetic field $B_{0}$, core current radius $a$, and background plasma density $\rho_{0}$.

In this form, the values of $\bar{I}, \bar{\delta}, \bar{\Delta}, \beta$, and $\gamma$ fully determine the evolution. For $\bar{\delta}, \bar{\Delta}=0.2, \beta=136$, and $\gamma=5 / 3$, numerical solution of Equation (9) shows that the peak velocity is reached early in the evolution and then quickly decays. For a constant current $\bar{I}(t)=\bar{I}_{c}$, the dynamics of the reverse current layer depends on how $\bar{I}_{c}$ compares to two critical values $\bar{I}_{e}$ and $\bar{I}_{s}$. When $\bar{I}_{c}=\bar{I}_{e}=\pi(\bar{\delta}+2) \sqrt{3(\bar{\Delta}+1)(1+\beta) /(2 \bar{\delta}+3)} \sim 83, \bar{b}(t)$ stays stationary, i.e., $\ddot{\bar{b}}=0$ and $\dot{\bar{b}}=0$ at $\bar{b}=1$. Below this value, the reverse current layer radially collapses inward due to insufficient internal magnetic pressure to balance out the external one. Above this value, the current piston pushes out the plasma and drives the compressional front. The internal magnetic pressure is initially larger than the external one and decreases as the piston expands. Let the two pressures be equal at $\bar{b}=\bar{b}_{0}$; at that location, $\dot{\bar{b}}$ reaches maximum $(\ddot{\vec{b}}=0)$. When $\bar{I}_{c}=\bar{I}_{s}=\pi\left(2 \bar{b}_{0}+\bar{\delta}\right)$ $\sqrt{3\left(\bar{b}_{0}+\bar{\Delta}\right)(3+(\gamma+1) \beta) /\left(3 \bar{b}_{0}+2 \bar{\delta}\right)} \sim 161$, the maximum speed of piston exceeds the normalized magnetosonic speed, $v_{\mathrm{ms}} / v_{\mathrm{A}}=\sqrt{1+\left(c_{s} / v_{\mathrm{A}}\right)^{2}}=\sqrt{1+\gamma \beta / 2}$, and the piston generates a traveling shock wave, i.e., $\ddot{\vec{b}}=0$ and $\dot{\bar{b}}=\sqrt{1+\gamma \beta / 2}$ at $\bar{b}=\bar{b}_{0} \cdot \dot{\bar{M}}=2 \pi(\bar{b}+\bar{\Delta}) \dot{\bar{b}}$ is used for the third term of the right side of Equation (9). Numerical solution shows that $\bar{b}_{0} \sim 1.3$ for $100<\bar{I}_{c}<200$. Given the expression for the total mass of the density shell, the compression ratio can be expressed as $X=\bar{\rho}=\left((\bar{b}+\bar{\Delta})^{2}-1\right) /\left((\bar{b}+\bar{\Delta})^{2}-\bar{b}^{2}\right)$.

This hybrid model exhibits both wave and pseudo-wave characteristics. Since the feature motion is determined by physical forces rather than by a dispersion relation, the layer will move at a range of possible speeds depending on the driving current and the local background conditions. The model also generates shocks for initial conditions (namely $\bar{I}_{c}>\bar{I}_{S}$ ) where the driving velocity exceeds the magnetosonic speed at early times. Thus, if $\bar{I}_{e}<\bar{I}_{c}<\bar{I}_{s}$ the phenomenon will be wavelike and sub-Alfvénic, whereas if $\bar{I}_{c}>\bar{I}_{s}$ the phenomenon will be shock-like and super-Alfvénic. This range of possible behaviors from low speed (sub-Alfvénic) to high speed (shock) satisfies the major observational constraint that sometimes subAlfvénic wave-like behavior is observed and sometimes, as manifested by Type II radio bursts, fast shock-like behavior is observed.

\section{Arched Flux Rope Experiment}

The theory in the previous section is motivated by measurements of a plasma flux rope experiment. This experiment was 


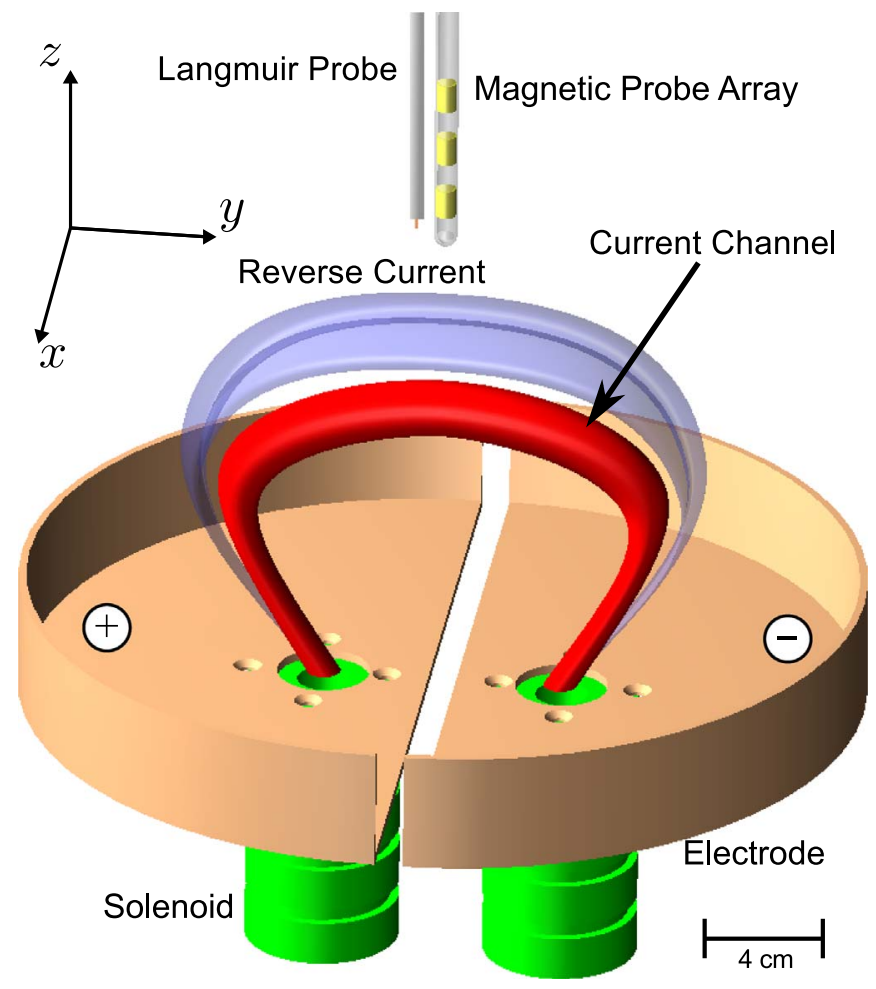

Figure 3. Diagram of an experimental apparatus showing electrodes, solenoids that generate a bipolar background field, and two nozzles on the electrodes for gas supply. A typical flux rope is shown in red, with the associated reverse current layer shown in blue. The Langmuir probe and magnetic probe array are located along the $z$-axis.

designed to produce an arched flux rope with dimensionless parameters similar to those of solar prominences $\left(\mu_{0} L v_{\mathrm{A}} / \eta \gg 1\right.$, $\beta \sim 0.1$ inside the loop and $\beta \sim 100$ in the background) (Stenson \& Bellan 2012; Ha \& Bellan 2016; Wongwaitayakornkul et al. 2017). This dimensionless equivalence allows solar prominences to be simulated in the lab with high repetition and control. A description of free parameters and constraints on dimensionless scaling in MHD is given in Ryutov et al. (2000); this shows that the experiment can be readily scaled to solar situations.

The experiment combines three subsystems to generate flux ropes: solenoids to provide a background bipolar magnetic field, gas ports to supply neutral argon gas, and electrodes which drive current through the plasma (Figure 3). The resulting arched flux rope then expands outward due to the hoop force. For a more detailed description of the experimental apparatus see Stenson \& Bellan (2012), Ha \& Bellan (2016), and Wongwaitayakornkul et al. (2017).

The experiments described in this paper use an additional hydrogen background prefill $\left(n \sim 10^{21} \mathrm{~m}^{-3}\right)$ not present in previous experiments (Stenson \& Bellan 2012; Ha \& Bellan 2016; Wongwaitayakornkul et al. 2017). This background gas serves two purposes: it creates a background hydrogen plasma for the flux rope to interact with and it enables distinguishing the motion of the flux rope (Ar) and background plasma $(\mathrm{H})$ from each other through spectroscopic filtering of images. This technique is exhibited in the left halves of the image sequence shown in Figure 4. This sequence shows the formation and propagation of a density front in the background hydrogen plasma. This layer (cyan) propagates ahead of the flux rope (red) with an increasing separation over time.

A fast ICCD movie camera, a magnetic probe array, and Langmuir probes are used to diagnose spatial and temporal characteristics of the plasma. The optically filtered fast ICCD camera provides the series of spectroscopic images shown in the left half of Figure 4. The magnetic probe array measures the magnetic field along the $z$-axis from voltages induced by changing magnetic fields. Assuming that the internal evolution is much slower than the global expansion, the time evolution can be converted (Haw et al. 2018) to spatial position with $\partial / \partial z \leftrightarrow v_{z}^{-1} \partial / \partial t$, with $v_{z}=15 \mathrm{~km} \mathrm{~s}^{-1}$. Then $J_{y}=(\boldsymbol{\nabla} \times \boldsymbol{B})$. $\hat{y} / \mu_{0}=\left(\partial_{z} B_{x}-\partial_{x} B_{z}\right) / \mu_{0} \approx\left(\partial_{t} B_{x}\right) /\left(\mu_{0} v_{z}\right)$, because the spatial variation of the flux rope is mostly in the $z$ direction $\left(\partial_{z} B_{x} \gg \partial_{x} B_{z}\right)$. Negatively biased Langmuir probes measured ion saturation current from the plasma at a given time along the $z$-axis. The ion density can be inferred from ion saturation current $I_{\text {sat }}$, with an isothermal assumption, i.e., $n_{i}=I_{\mathrm{sat}} /\left(0.6 e A c_{s}\right) \quad$ with $c_{s}=\sqrt{2 \kappa T_{e} / m_{i}}=20 \mathrm{~km} \mathrm{~s}^{-1}$ and probe tip area $A=7.6 \times 10^{-6} \mathrm{~m}^{2}$.

The solid lines in Figures 5(a) and (c) are example plots of the measured time dependence of probes at three different values of $\bar{z}$. This data was fitted with analytic functions that are plotted as dashed lines in Figures 5(a) and (c). $\bar{J}_{y}(\bar{t})$ was modeled as two Gaussian peaks and $\bar{\rho}(\bar{t})$ was modeled as a piecewise function that increases linearly and decreases exponentially. The Langmuir probe data was taken from 164 shots at 24 different locations $(\bar{z}=10-30)$. The magnetic data were taken from 83 shots at 4 different locations $(\bar{z}=16-22)$. Spacetime contour plots of $\bar{J}_{y}(\bar{z}, \bar{t})$ and $\bar{\rho}(\bar{z}, \bar{t})$ were constructed from the measurements and are shown in Figures 5(b) and (d). The positive and negative component of $\bar{J}_{y}$ contour are scaled differently to enhance the negative component. A detailed description of the method for construction of these contour plots is given in the Appendix. These measurements, along with the lower brightness of optical line emission in between the two opposite current features from the images, imply that there is a depletion in density in the region between the two opposite currents. The Langmuir probe signals show a clear jump in density indicating a shock in ion density with thickness $\delta=0.8 \pm 0.3 \mathrm{~cm}$ and compression ratio $X=$ $1.8 \pm 0.4$. Let $t_{0}$ and $t_{1}$ be the time in which Langmuir probe data first increase from the background level and reach the peak respectively. Then $\delta$ is deduced from the difference in the arrival times and from the speed of the feature as $\delta=v_{z}\left(t_{1}-t_{0}\right)$. $X$ is determined by the ratio of the signal at those two times, $X=\bar{\rho}\left(t_{1}\right) / \bar{\rho}\left(t_{0}\right)$.

The following section describes results from a 3D ideal MHD numerical simulation of the experiment. This numerical simulation confirms that the reverse current mechanism can generate shocks and also allows a shock analysis with high spatial resolution.

\section{MHD Numerical Simulation}

The 3D ideal MHD simulation of the experiment was performed on the Los Alamos Turquoise cluster as part of the Los Alamos COMPutational Astrophysical Simulation Suite to reproduce the Caltech solar loop experiment (Li \& Li 2003; Zhai et al. 2014; Haw et al. 2018). The simulation follows the evolution of eight parameters: mass density $\rho$, pressure $p$, velocity $\boldsymbol{v}$, and magnetic field $\boldsymbol{B}$ inside a numerical Cartesian box of size $32 a$. The initial parameters are set to emulate the 

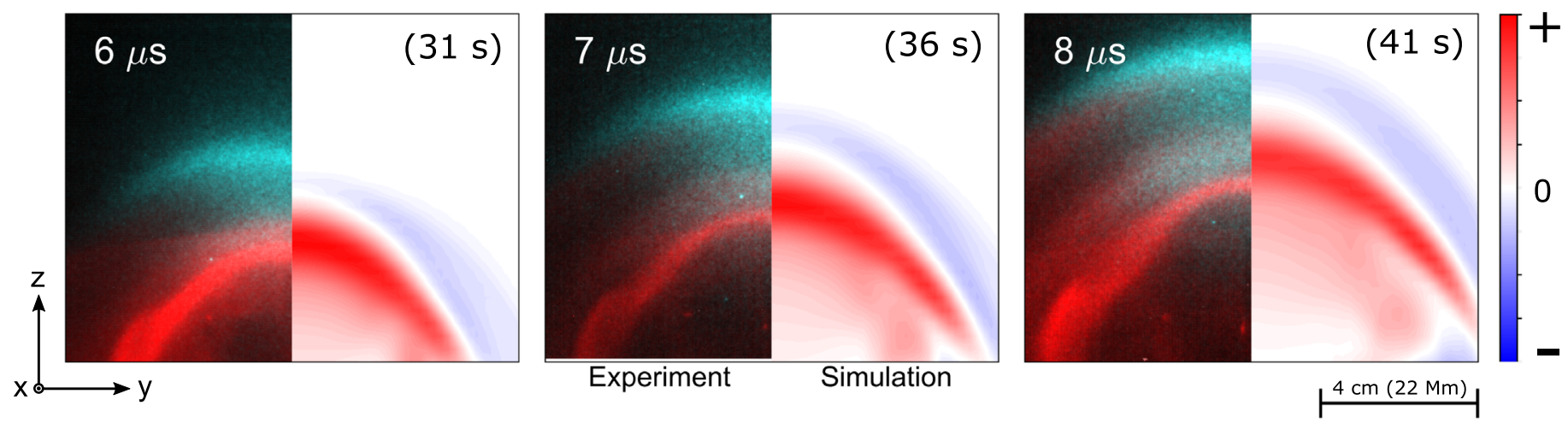

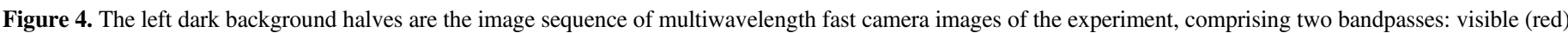

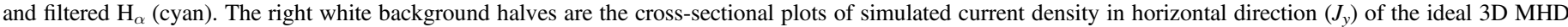

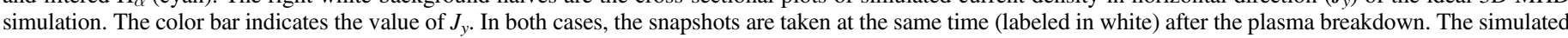

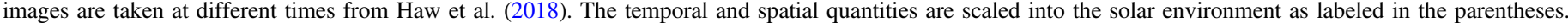

experimental setup shown in Figure 3(a). The initial density consists of two conic-shape density profiles as produced by the gas nozzles at the footpoints when there is a substantial prefilled uniform background density. The initial magnetic field is the bipolar potential field from the two solenoids behind the electrodes. The pressure is calculated from the isothermal assumption and the plasma is initially at rest. Electric current is injected into the system by adding azimuthal magnetic field corresponding to a group of circular current loops (Simpson et al. 2001; Wongwaitayakornkul et al. 2017; Haw et al. 2018) with a sinusoidal time dependence that mimics that in the experiment. Figure 4 shows the time dependence of the loop expansion as observed in the experiment (left halves of figures) and as calculated in the simulation (right halves of the figures). The simulation is in reasonable agreement with the laboratory plasma in terms of the loop's evolution and magnetic field. The full diagnostic capability of the simulation allows for more detailed analysis than possible in the experiment of the temporal and spatial dependence of the main current channel (red) and the reverse current layer (blue).

The evolution of the system can be broken down into three stages: formation, driving, and decoupling. Figure 6 shows the evolution of the apex, defined as the $z$ coordinate of local maxima of $\left|J_{y}\right|$. Let $\bar{t}=v_{\mathrm{A}} t / a$ and $\bar{z}=z / a$, where $a$ is the minor radius of the flux rope and the nominal length for normalization and $v_{\mathrm{A}}$ is the Alfvén speed. In the formation stage $(\bar{t}<0.22)$, a current flowing in the positive $y$ direction is injected into the system. The curved current channel expands in major radius due to its hoop force and compresses in the positive $z$ direction, forming a flattened current channel flowing mainly in the positive $y$ direction. Later in the driving stage $(0.22<\bar{t}<0.40)$, a reverse current layer is formed above the original expanding current channel; this reverse current is formed to satisfy the magnetic flux conservation condition in the stationary conducting background plasma above the current channel. The increased azimuthal magnetic field in between the main and reverse currents pushes the reverse current layer out radially and leaves behind a region of depleted density (region between the red and blue regions in Figure 3(a) and in Figure 4). The plasma boundary, or contact front (bottom of the blue region in Figure 4), acts as a magnetic piston, compressing the swept-out plasma. With sufficiently strong current, the piston expands faster than the magnetosonic speed in the plasma. As a result, a shock is formed ahead of the piston and all the mass swept up by the shock is compressed into a thin layer immediately behind the shock. The current layer and the shock are indistinguishable at this stage. The thin current layer is repelled by the core current and quickly reaches the equilibrium standoff distance as prescribed by Equation (9). The full dynamics of the induced current sheet are governed by the force balance of $f_{e}$ and $f_{c}$, described in Section 2.2. At $\bar{t} \sim 0.40$, the current injection stops, causing the driver and the contact front to decelerate. With the condition described in Section 2.1, the contact front and the shock separate. The shock remains in motion, while the contact front decelerates with the driver. Thus, for $\bar{t}>0.40$ the shock is decoupled from the piston and propagates freely.

The analytic theory presented in Section 2 used the cylindrical coordinate system shown in Figure 2. In this section we present a result of a numerical simulation where Cartesian coordinates are used instead. The correspondence follows $\{r \rightarrow z, y \rightarrow y, \theta \rightarrow x\}$. Since the apex moves in the $z$ direction, it is convenient to plot the parameters along the $z$-axis. Figure 7(a) plots contours of $\bar{J}_{y}$ as a function of $\bar{z}$ and $\bar{t}$. Recall that $\bar{J}_{y}$ is the horizontal component of the current at the apex as shown in Figure 3(a). Figure 7(a) shows the main current channel in red and the reverse current in blue slightly to the right of the red. The experimental $\bar{J}_{y}$ contour in Figure 3(b) shows a larger decay of amplitude in $\bar{z}$ compared to its simulated counterpart in Figure 7(a). This is expected due to a higher resistivity in the experiment. The slope, $d \bar{t} / d \bar{z}$, of the reverse current layer in Figure 7(a) implies a propagation speed $\bar{v}_{s}=d \bar{z} / d \bar{t}=25$, which exceeds the local fast magnetosonic speed $\bar{v}_{m s}=10$ of the unshocked plasma.

The transition is therefore a fast-mode shock and should obey the RH relations given in Section 2.1. Figure 7(b) plots the temperature $\bar{T}$, showing that the loop becomes cooler as its size increases. This is expected from the adiabatic equation of state. The main current channel, however, remains the hottest part even after the shock forms. The density $\bar{\rho}$, in Figure 7(c), shows that the density amplification is largest at the location of the shock front. Behind the shock, the density depletes to a value lower than the background (black region). Contrary to the experiment (Figure 3(c)), the simulated density contour (Figure 7(c)) appears to contain a single peak at most times. A two-peak profile can only be observed when the shock is initially formed, i.e., $\bar{t} \sim 0.56$. The density peak of the main current decays abruptly, while the density peak of the shock 

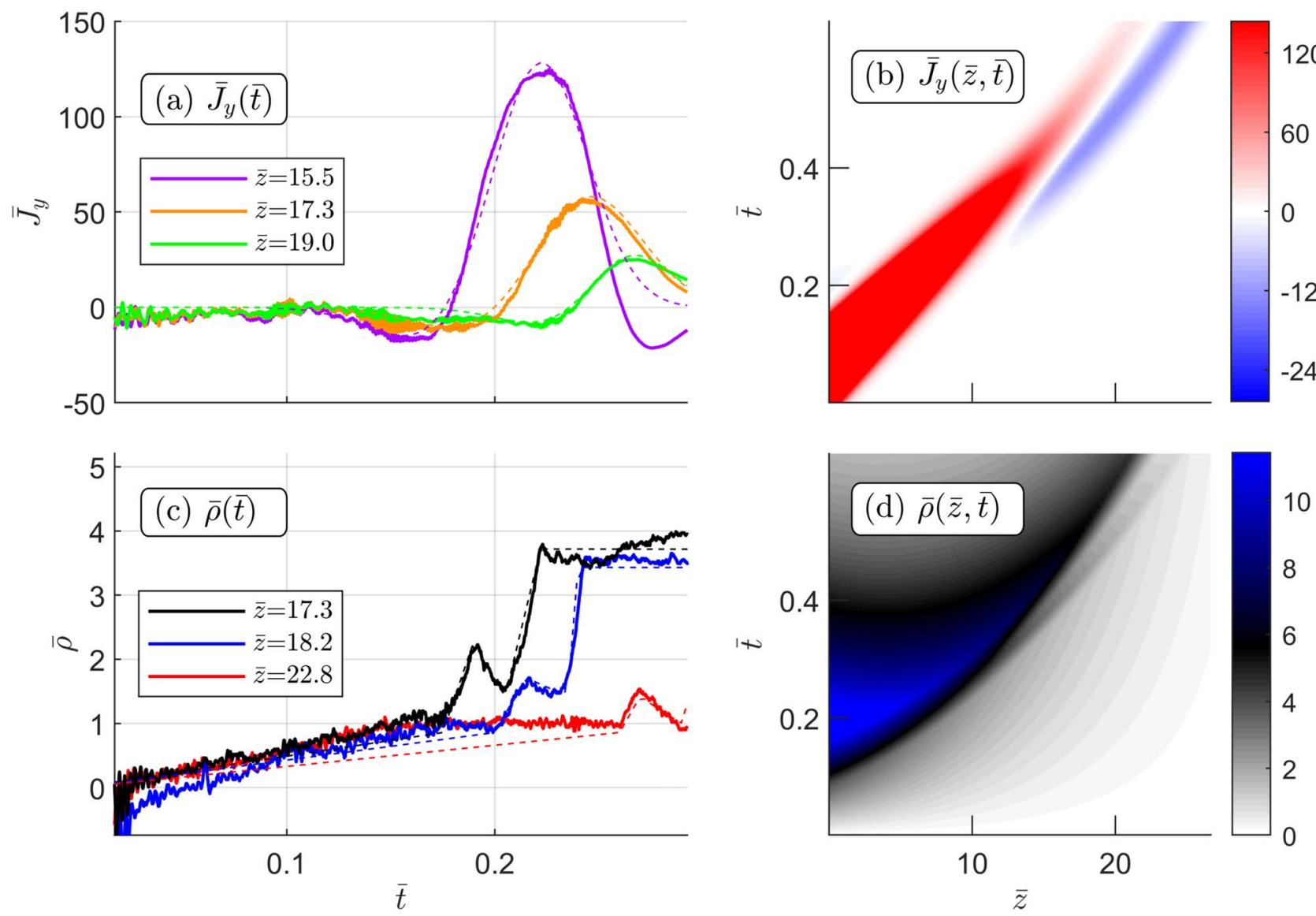

Figure 5. (a) and (c) show the experimental sample time-series of the magnetic and Langmuir probes (solid line) with the reconstructed fitted profiles (dashed line). (b) and (d) plot the spacetime profiles of the dashed line of $\bar{J}_{y}$ and $\bar{\rho}$ in (a) and (c). The normalized parameters for the experiment are $a=1.1 \mathrm{~cm}, v_{\mathrm{A}}=0.43 \mathrm{~km} \mathrm{~s}{ }^{-1}$, $\tau=a / v_{\mathrm{A}}=130 \mu \mathrm{s}, \rho_{0}=9.1 \times 10^{-9} \mathrm{~kg} \mathrm{~m}^{-3}, B_{0}=0.46 \mathrm{G}$, and $J_{0}=\sqrt{\rho_{0} / \mu_{0}} / \tau=3.3 \mathrm{kA} \mathrm{m}^{-2}$. These parameters can be scaled to the solar context using $a=6.25 \mathrm{Mm}, v_{\mathrm{A}}=41.7 \mathrm{~km} \mathrm{~s}^{-1}, \tau=135 \mathrm{~s}, \rho_{0}=7.0 \times 10^{-12} \mathrm{~kg} \mathrm{~m}^{-3}, B_{0}=1.2 \mathrm{G}$, and $J_{0}=16.7 \mu \mathrm{A} \mathrm{m}^{-2}$. The method for scaling to the solar context is given in Haw et al. (2018) and in Ryutov et al. (2000).

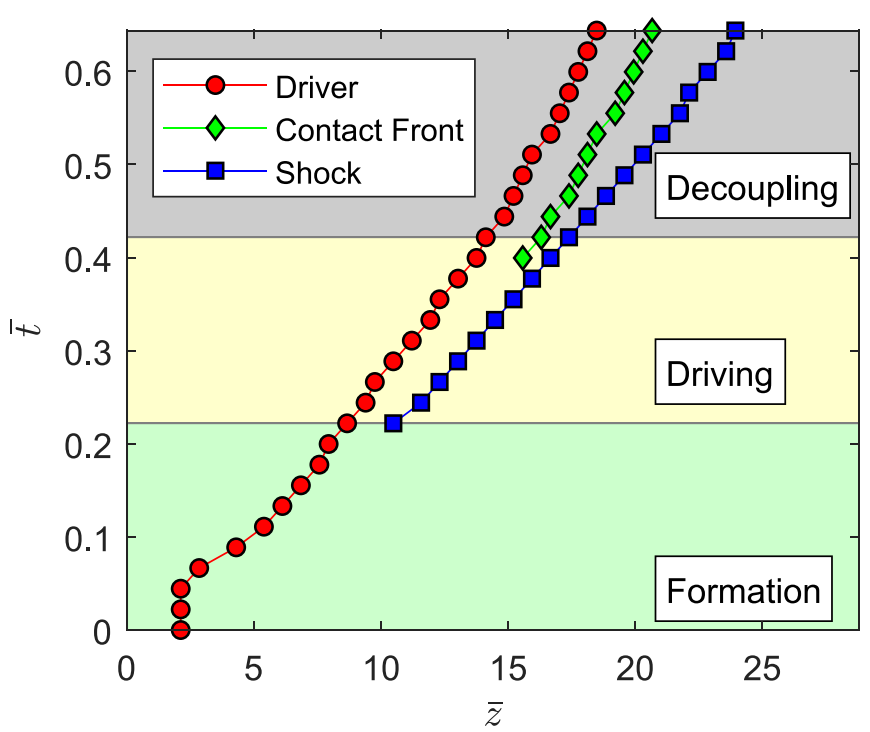

Figure 6. Evolution of apex locations of the simulation during the three stages.

remains more or less constant. As the system expands, the volume increases. However, only the shock acquires the additional material from the background plasma. In the experiment, the plasma behind the electrode most likely supplies material to sustain the density of the main current loop. Lastly, the velocity plot, shown in Figure 7(d), exhibits the initially fast expanding loop due to the strong flow of material behind the loop. At $\bar{t}<0.38$, the plasma behind the loop experiences a large magnetic force in the positive $z$ direction from the current injection indicated by its large $v_{z}$. For $\bar{t}>0.44$, the shock front and its plasma flow reach constant terminal speeds.

Figure 8 displays the set of quantities, in the frame moving with the shock of speed $\bar{v}_{s}$, on the shock boundaries at time $\bar{t}=0.56$ from the simulation; this time it is denoted as "shock" in Figure 7. $\bar{\rho}$ is the plasma density, $\bar{p}$ is the gas pressure, $\bar{v}_{z}$ is the velocity in $z$ direction, and $\bar{B}_{t}$ is the tangential magnetic field in the $x y$-plane. The flow speed in the shock frame is defined as $\bar{v}_{z}^{\prime}=\bar{v}_{s}-\bar{v}_{z}$. This relation is obtained by (i) moving to a shock frame $\bar{v}_{z} \rightarrow \bar{v}_{z}-\bar{v}_{s}$ and (ii) flipping the sign $\bar{v}_{z}-\bar{v}_{s} \rightarrow \bar{v}_{s}-\bar{v}_{z}$ to have $\bar{v}_{z}^{\prime}$ be positive in the $-\hat{z}$ direction. The subscripts $u$ and $d$ represent the upstream and downstream value of the parameters, which are taken from the boundary of the shocks denoted by the vertical dashed lines in Figure 8 . Given that the fast magnetosonic speed is always greater than the Alfvén speed $v_{\mathrm{ms}}=\sqrt{v_{\mathrm{A}}^{2}+c_{s}^{2}}>v_{\mathrm{A}}$, the background colors specify three regions in velocity phase space based on $v_{z}^{\prime}$ : (i) $v_{z}^{\prime}>v_{\mathrm{ms}}$, (ii) $v_{\mathrm{ms}}>v_{z}^{\prime}>v_{\mathrm{A}}$, and (iii) $v_{z}^{\prime}<v_{\mathrm{A}}$. The shock takes the plasma flow from region (i) to (ii), which is the characteristic of the fast magnetosonic type. 


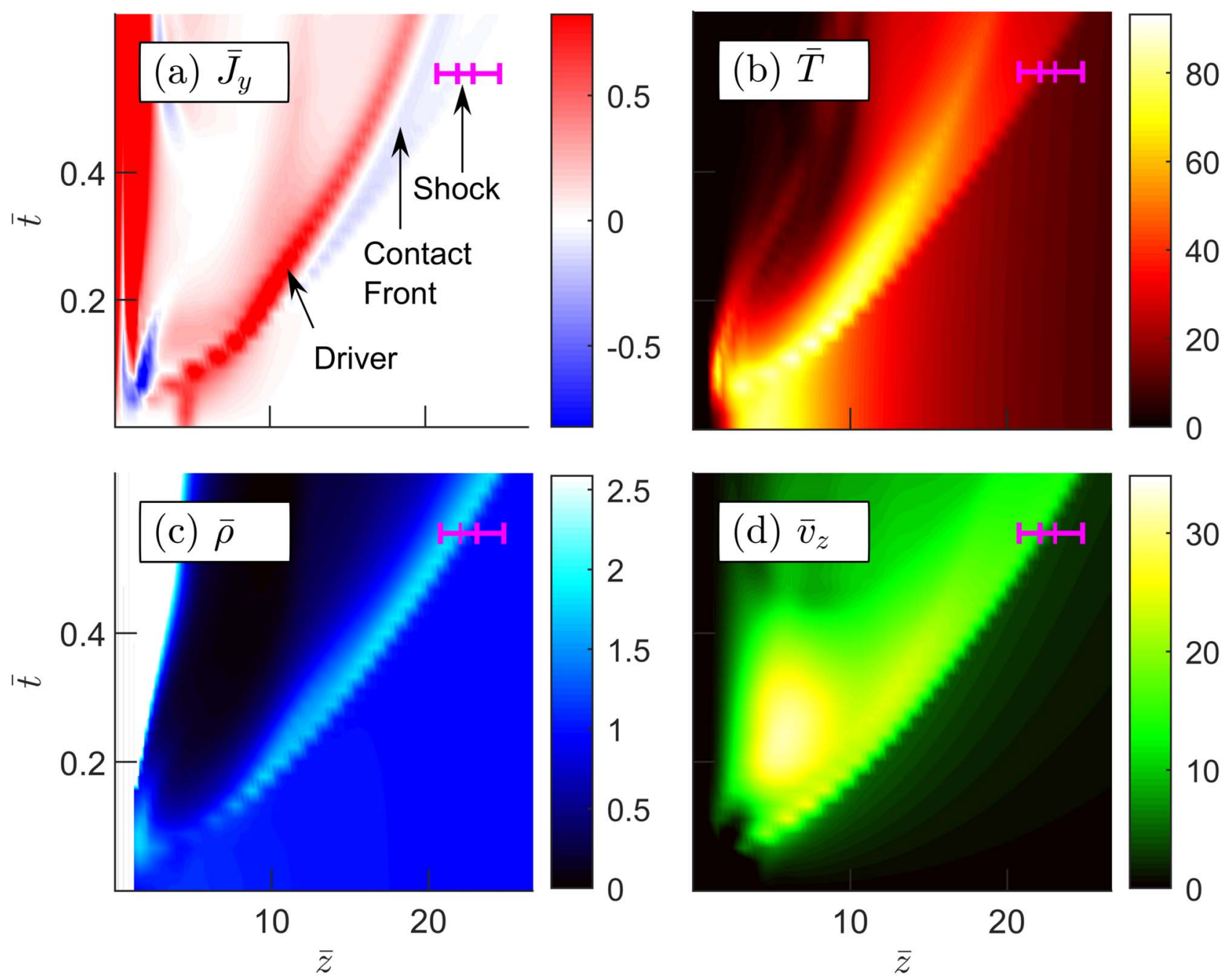

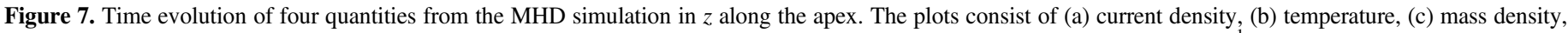

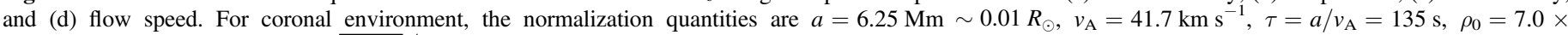
$10^{-12} \mathrm{~kg} \mathrm{~m}^{-3}, B_{0}=1.2 \mathrm{G}, J_{0}=\sqrt{\rho_{0} / \mu_{0}} / \tau=16.7 \mu \mathrm{A} \mathrm{m}^{-2}$, and $T_{0}=m_{H} v_{\mathrm{A}}^{2} / 2 \kappa=9.7 \times 10^{4} \mathrm{~K}\left(m_{\mathrm{H}}=1 \mathrm{u}\right)$.

Given the characteristic of the background plasma $\mathcal{M}$ and $\beta$, we may now calculate the expected compression ratios $X$ and $Y$ using Equations (5) and (6). For the simulation at the time $\bar{t}=0.56$, the compression ratios can be determined from the upstream parameters $\bar{\rho}_{u}=1, \bar{v}_{z, u}^{\prime}=1, \bar{B}_{t, u}=1, \bar{p}_{u}=1$, which gives $\mathcal{M}=1.8, \beta=136$. Using these values in Equations (5) and (6) gives $X=2.1$ and $Y=3.8$, as indicated with diamond markers in Figure 8. The simulated compression ratios are $\bar{\rho}_{d} / \bar{\rho}_{u}=1.8, \bar{v}_{z, u}^{\prime} / \bar{v}_{z, d}^{\prime}=2.1, \bar{B}_{t, d} / \bar{B}_{t, u}=1.9$, and $\bar{p}_{d} / \bar{p}_{u}=3.9$, where upstream and downstream values are measured at the locations of the vertical dashed lines. These compression ratios are also consistent with the analytic expression in Section 2.2. The simulated compression ratios match well with the analytic expression for $X=\bar{\rho}_{d} / \bar{\rho}_{u}$ and $Y=\bar{p}_{d} / \bar{p}_{u}$, indicating that the perturbation front is a fast magnetosonic shock. For $\mathcal{M}=1.8$, $\beta=136$, and $\gamma=5 / 3$, the magnetosonic mach number $\mathcal{M}_{\mathrm{ms}}=\mathcal{M} / \sqrt{1+2 / \gamma \beta}>1$. The normalized entropy, $\bar{S}=$ $\ln \left(\bar{p} / \bar{\rho}^{\gamma}\right)$, also increases across the shock $(\Delta \bar{S}=0.4)$.

The simulation shows that a flux rope erupted from the injection of the electrical current induces a layer of reverse current that acts as a magnetic piston. Impulsive expansion of the piston produces a fast-mode shock that is also a current layer due to the compression of the background magnetic field. The dynamics of the piston is described in Section 2.2 and is compared to the simulation in Haw et al. (2018). In the driving phase, the shock and piston are attached. Thus, the shock evolution is governed by the movement of the driver, displaying the characteristic of a pseudo-wave. In the decoupling stage, the shock detaches from the piston because the piston decelerates to a speed below the local fast magnetosonic wave speed. The piston and shock separate and so the shock escapes the driving influence of the piston. The shock is then self-propagating, representing the wave behavior. The presence of both the wave and pseudo-wave behavior of the simulated shock supports a hybrid theory of CME-driven EUV wave.

\section{Discussion}

Despite the variety of different models to explain EUV waves, the physical manifestation of all proposed models is a propagating, compressive current layer. This current layer is 


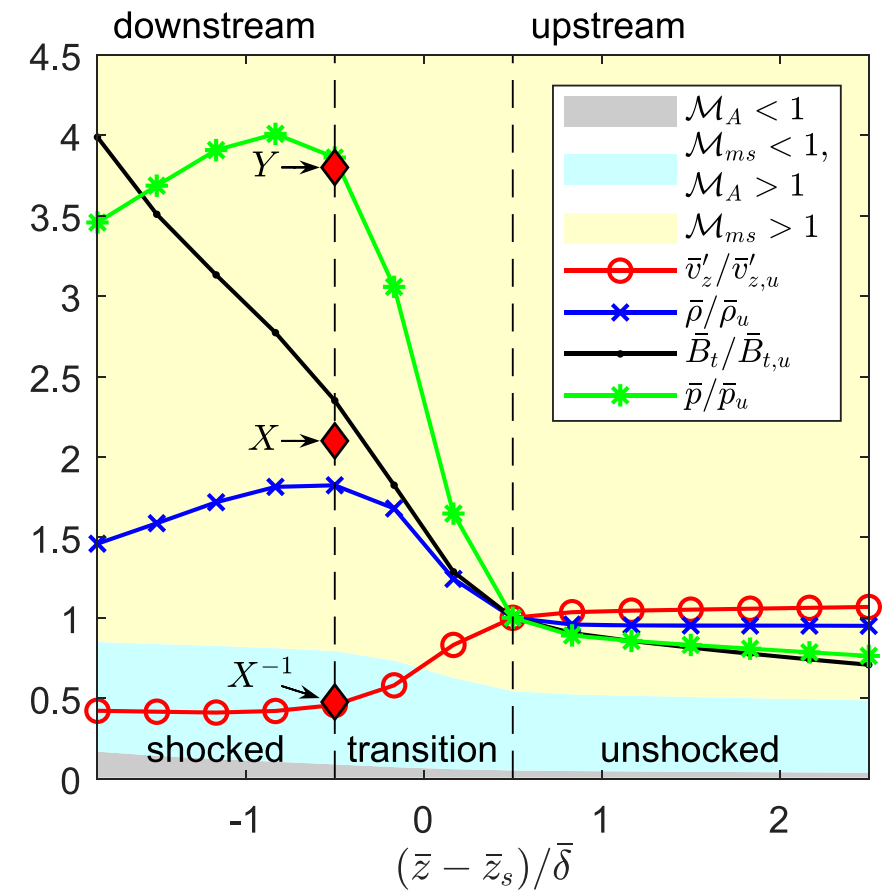

Figure 8. Transition of four variables in the MHD simulation: flow velocity in the shock frame $\bar{v}_{z}^{\prime}=\bar{v}_{s}-\bar{v}_{z}$, mass density $\bar{\rho}$, pressure $\bar{p}$, and tangential magnetic field $\bar{B}_{t}$ across the shock. The $\bar{z}$ range and time for this plot are shown as the magenta line in Figure 7 . The background colors represent the three regions in velocity phase space: (i) $v_{z}^{\prime}>v_{\mathrm{ms}}$, (ii) $v_{\mathrm{ms}}>v_{z}^{\prime}>v_{\mathrm{A}}$, and (iii) $v_{z}^{\prime}<v_{\mathrm{A}}$. The two vertical dashed lines indicate the boundaries of the shock. Each parameter is normalized to their upstream value. The expected compression ratios are labeled $Y, X$, and $X^{-1}$ on the downstream boundary. $\bar{z}_{s}$ is the location of shock center and $\bar{\delta}$ is the shock thickness.

caused by compression of the background magnetic field, but in previous models this current was not identified as being in the reverse direction with respect to the current in the erupting flux rope. Fast-mode pulses/shocks are, by definition, compressive current layers. The current layer model in Delannée et al. (2008) was previously characterized purely in terms of the electric current; however, it is also cospatial with a compressive density pulse. The wake model in Chen et al. (2002) identifies the EUV wave as a compressive front but does not take into account that a compressional front must necessarily contain electric current. Finally, the successive magnetic reconnection model in Attrill et al. (2007) creates an expanding density enhancement with electric current. This is not implying that all the models are equivalent, but instead highlights the overlap of the differences. In some cases, such as the current layer (Delannée et al. 2008) and wake models (Chen et al. 2002), it is not clear if there is a physical distinction between these two models (Long et al. 2017). Table 1 in Long et al. (2017) presented predictions of pulse physical properties from six previous theories and Table 2 of Long et al. (2017) showed how these predictions compare to observations. Table 1 of the present paper shows the predictions given by the model presented in this paper for the properties listed in Long et al. (2017). $v_{\mathrm{CME}_{\perp}}$ and $a_{\mathrm{CME}_{\perp}}$ represent the lateral velocity and acceleration of the erupting CME. $A_{\mathrm{CME}}$ represents the area bounded by the CME bubble. These predictions are similar to the previous fast-mode wave/shock and current shell theory, which are in good agreement with observations. The physical manifestation of the pulse would then be dictated by whether the time of observation is in the driving or decoupling phase of the event.

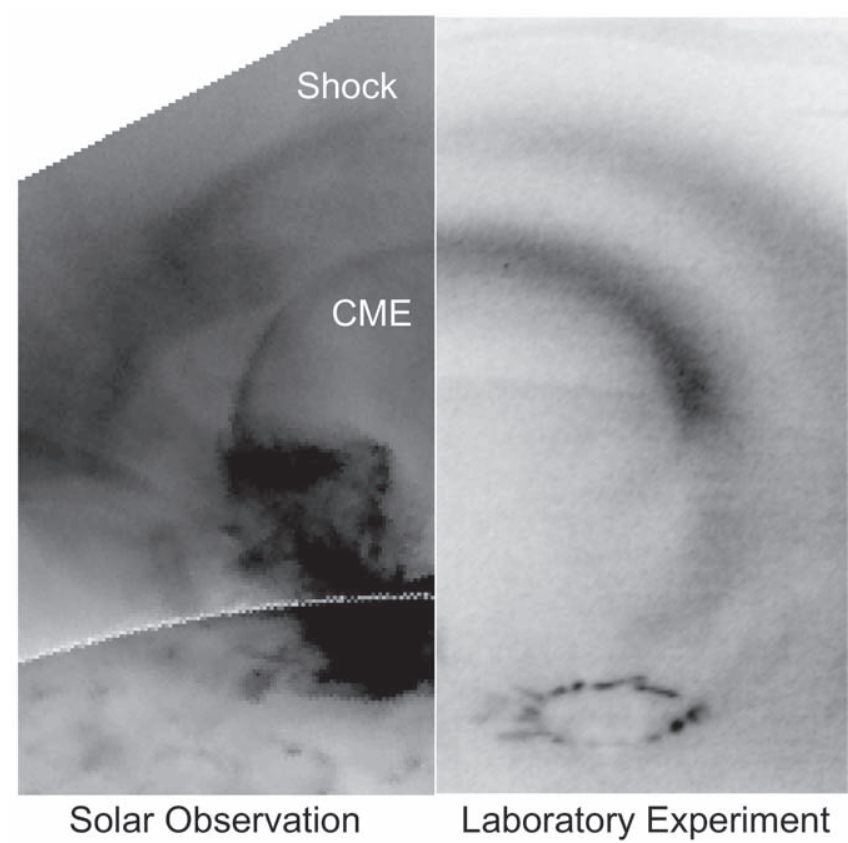

Figure 9. Comparison of (left) the low coronal EUV (193 $\AA$ ) shock wave observed with AIA/SDO on 2010 June 13 (courtesy of NASA/SDO and the AIA, EVE, and HMI science teams; it is the same image as used in Ma et al. 2011) and (right) visible emission from the Caltech experiment in reverse grayscale.

Table 1

Prediction from This Model for the Properties Listed in Long et al. (2017)

\begin{tabular}{|c|c|c|c|}
\hline \multirow{3}{*}{ Pulse Properties } & \multicolumn{3}{|c|}{ This Hybrid Model } \\
\hline & \multirow{2}{*}{ Driving Phase } & \multicolumn{2}{|c|}{ Decoupling Phase } \\
\hline & & $\begin{array}{l}\text { Small amp. } \\
\text { Linear Wave }\end{array}$ & $\begin{array}{l}\text { Large amp. } \\
\text { Wave/Shock }\end{array}$ \\
\hline Phase velocity $[v]$ & $\gtrsim v_{\mathrm{CME}_{\perp}}$ & $v_{\mathrm{ms}}$ & $>v_{\mathrm{ms}}$ \\
\hline Acceleration $[a]$ & $\gtrsim a_{\mathrm{CME}_{\perp}}$ & $\begin{array}{c}>v_{\mathrm{CME}_{\perp}} \\
0\end{array}$ & $\begin{array}{c}>v_{\mathrm{CME}_{\perp}} \\
<0\end{array}$ \\
\hline Broadening & $f\left(a_{\mathrm{CME}_{\perp}}\right)$ & $\approx 0$ & $>0$ \\
\hline$\Delta B$ & $>0$ & $>0$ & $>0$ \\
\hline$\Delta T$ & Adia. $+Q_{\text {Joule }}$ & Adia. & Adia. $+Q$ \\
\hline$\Delta n_{e}$ & Compression & Compression & Compression \\
\hline Height & $f($ CME bubble $)$ & $f\left(B, n_{e}\right)$ & $f\left(B, n_{e}\right)$ \\
\hline Area bounded & $A_{\mathrm{CME}}$ & $>A_{\mathrm{CME}}$ & $>A_{\mathrm{CME}}$ \\
\hline Rotation & Possible & Possible & Possible \\
\hline Reflection & No & Yes & Yes \\
\hline Refraction & No & Yes & Yes \\
\hline Transmission & No & Yes & Yes \\
\hline Stationary fronts & Yes & Yes & Yes \\
\hline Cospatial Type II & No & No & Possible \\
\hline Moreton wave & No & No & Possible \\
\hline
\end{tabular}

The hybrid model proposed here is consistent with existing observations. The main observation supporting the fast-mode shock model is the strong correlation between the type II radio burst and the EUV waves (Klassen et al. 2000; Biesecker et al. 2002; Vršnak \& Cliver 2008). Assuming the density profile of the corona, one could deduce the speed of the emitter from the radio signals, which turns out to be faster than the coronal Alfvénic speed, suggesting that the emitter is a coronal shock front. In addition, CMEs are strongly correlated with the observation of EUV waves (Biesecker et al. 2002; Warmuth et al. 2005; Veronig et al. 2008). Figure 9 shows a system of 


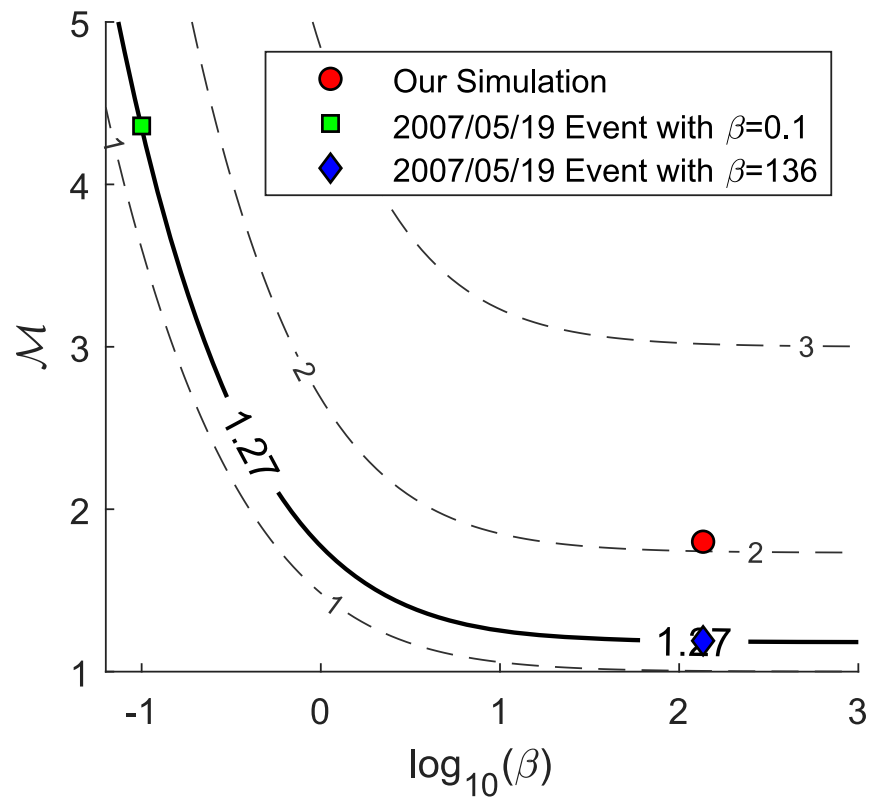

Figure 10. Contour of the compression ratio $X(\mathcal{M}, \beta)$ as determined by Equation (7). The compression ratio of the 2007 May 19th event in Muhr et al. (2011) $X=1.27$ is plotted as a solid line. The two points corresponding to $\beta=0.1$ and 136 along $X=1.27$ are plotted with green and blue markers. The value used in the simulation of this work is plotted with a red marker.

flux rope and shock for both an AIA observation on 2010 June 13 and our laboratory experiment in reverse grayscale (i.e., black is the maximum value and white is the minimum value). We have shown that an erupting flux rope can generate a fast magnetosonic shock. The fast magnetosonic shock generated by an erupting CME should then be able to produce the type II radio burst.

Previously measured compression ratios of the observed global EUV wave, deduced from the intensity ratios of line emission, are 1.05-1.30 (Muhr et al. 2011; Zhukov 2011; Long et al. 2015). In the numerical MHD simulation shown in Figures $6-8$, we obtain the compression ratio of $X=1.9$, which is different from the observed values due to the different $\mathcal{M}$ and $\beta$ of each event; Equation (7) shows the relation between $X, \mathcal{M}$, and $\beta$. For example, substituting $\mathcal{M}=4.36$ (note that $\mathcal{M}=\mathcal{M}_{\mathrm{ms}} \sqrt{1+2 / \gamma \beta}$ ) and $\beta=0.1$ into Equation (7) gives $X=1.27$, as presented for the 2007 May 19 event in Muhr et al. (2011). While a uniform $\beta=0.1$ is commonly used at $z \sim 70-200 \mathrm{Mm}\left(0.1 R_{\odot}-0.2 R_{\odot}\right.$ Ma et al. 2011; Long et al. 2015) above the solar surface as suggested by Gary (2001), a more recent simulation (Bourdin 2017) proposed that a varying $\beta=20-200$ is more suitable in that region. This range of $\beta$ is used in our simulation. If we were to use $\mathcal{M}=1.19$ and $\beta=136$, the corresponding $X$ according to Equation (7) would also be $X=1.27$. Figure 10 shows the contour of compression ratio $X$ in $(\mathcal{M}, \beta)$-space. $X=1.27$ is drawn with a solid line. The two points $(\mathcal{M}=4.36, \beta=0.1)$ and $(\mathcal{M}=1.19, \beta=136)$ both correspond to $X=1.27$ and are plotted with green and blue markers, respectively. For $\beta \gg 10, X$ is no longer sensitive to $\beta$ and the shock approaches the hydrodynamic limit. While $\beta$ and $v_{\mathrm{ms}}$ are the properties of the background plasma, the expanding speed of the structure depends on the internal current of the CME's core, as discussed in Section 2.2. Consequently, the observation of the global EUV waves and their measured compression ratios could give us insight into the eruption mechanism of the CME.
The reverse current piston can produce both waves and shocks, depending on the driving and observing timescale. When the piston moves at a speed below the fast magnetosonic speed of the background plasma $\left(v_{\text {piston }}<v_{\mathrm{ms}}\right)$, the piston drives a simple wave that can eventually steepen into a shock. When $v_{\text {piston }}>v_{\mathrm{ms}}$, a shock forms due to the piston effect. In this case, the shock evolves in two stages: (i) attached to the driver and then (ii) decoupled from the driver. Depending on the time of the observation, the compressional front can behave as a wave or a pseudo-wave. The shock exhibits pseudo-wave behavior and wave behavior in stages (i) and (ii), respectively. The analytic model can be used to predict the condition, time, and height in which the two stages occur. This can explain why EUV waves are sometime slower than the expected coronal fast-mode speed (Wills-Davey et al. 2007). Several observations had reported detecting two-component bright fronts with either sharp (Liu et al. 2010) or diffuse (Cohen et al. 2009; Cheng et al. 2012) outer structure, which could respectively correspond to shock (sharp) and wave (diffuse) perturbation driven by a bright sharp piston current layer. The magnetic piston decelerates late in the simulation and eventually reaches a stationary equilibrium where the internal magnetic pressure balances the external background pressure. This could be an explanation for the observed deceleration (Warmuth et al. 2004; Long et al. 2008; Veronig et al. 2008) and stationary brightenings (Ofman \& Thompson 2002; Terradas \& Ofman 2004). The existing observations, supporting wave/ pseudo-wave bimodality (Harra \& Sterling 2003; Patsourakos \& Vourlidas 2009; Chen \& Wu 2011; Ma et al. 2011), show the transition from pseudo-wave to wave as illustrated in Figure 6. Future observations of high-cadence detailed kinematics of the decoupling process would provide a rigorous test for this hybrid model.

In summary, a single description of EUV waves as compressional MHD perturbations reconciles the important features of previous models and is supported by a laboratory experiment and a numerical simulation. A magnetically induced reverse current layer produced by an erupting flux rope creates a single coherent pulse that can be observed as a wave, pseudo-wave, and shock, depending on the plasma parameters and driving/observing timescale. By applying this interpretation of the coronal pulse generating mechanism, EUV wave observations could be used to diagnose the internal structure of CMEs.

\section{Appendix \\ Experimental Contour Reconstruction}

The following steps are used to create the spacetime contour plots shown in Figures 5(b) and (d):

1. A single point measurement at location $\bar{z}=z_{j}^{*}$ and $z_{\rho}^{*}$ gives experimental time-series $\bar{J}_{y, \exp }(\bar{t})$ and $\bar{\rho}_{\text {exp }}(\bar{t})$, respectively.

2. A constructed time-series profile for both parameters are defined as two Gaussian peaks for $\bar{J}_{y \text {,con }}(\bar{t})$ and a piecewise function that increases linearly and decreases exponentially for $\bar{\rho}_{\text {con }}(\bar{t})$. The analytic expressions are given by Equations (10) and (11):

$$
\begin{aligned}
\bar{J}_{y, \text { con }}\left(\bar{t} ; J_{ \pm}, t_{ \pm}, \tau_{ \pm}\right)= & J_{+} \exp \left(-\frac{\left(\bar{t}-t_{+}\right)^{2}}{2 \tau_{+}^{2}}\right) \\
& -J_{-} \exp \left(-\frac{\left.\left(\bar{t}-t_{-}\right)\right)^{2}}{2 \tau_{-}^{2}}\right)
\end{aligned}
$$




$$
\begin{aligned}
& \bar{\rho}_{\text {con }}\left(\bar{t} ; \rho_{i}, t_{i}, \lambda\right)_{i=0,1,2,3} \\
& = \begin{cases}\left(\rho_{0} / t_{0}\right) \bar{t}, & \bar{t}<t_{0} \\
\left(\rho_{1}-\rho_{0}\right)\left(\bar{t}-t_{0}\right) /\left(t_{1}-t_{0}\right)+\rho_{0}, & t_{0}<\bar{t}<t_{1} \\
\rho_{1}\left(\rho_{2} / \rho_{1}\right)^{\left(\bar{t}-t_{1}\right) /\left(t_{2}-t_{1}\right)}, & t_{1}<\bar{t}<t_{2} \\
\left(\rho_{3}-\rho_{2}\right)\left(\bar{t}-t_{2}\right) /\left(t_{3}-t_{2}\right)+\rho_{2}, & t_{2}<\bar{t}<t_{3} \\
\rho_{3} \exp \left(-\left(\bar{t}-t_{3}\right) / \lambda\right), & \bar{t}>t_{3} .\end{cases}
\end{aligned}
$$

3. For a given pair of $\left(z_{j}^{*}, z_{\rho}^{*}\right)$, the comparison between the experimental and constructed profile gives fitting parameters for this particular pair of $\bar{z}$ :

$$
\begin{aligned}
& \left\{\begin{array}{c}
\bar{J}_{y, \exp }(\bar{t}) \leftrightarrow \bar{J}_{y, \text { con }}\left(\bar{t} ; J_{ \pm}, t_{ \pm}, \tau_{ \pm}\right) \\
\bar{\rho}_{\exp }(\bar{t}) \leftrightarrow \bar{\rho}_{\text {con }}\left(\bar{t} ; \rho_{i}, t_{i}, \lambda\right)
\end{array}\right\} \stackrel{\text { extract }}{\rightarrow} \\
& \left\{\begin{array}{c}
\left.J_{ \pm}, t_{ \pm}, \tau_{ \pm}\right\}, \text {at } \bar{z}=z_{j}^{*} . \\
\left.\rho_{i}, t_{i}, \lambda\right\}, \text { at } \bar{z}=z_{\rho}^{*} .
\end{array}\right.
\end{aligned}
$$

4. The pair $\left(z_{j}^{*}, z_{\rho}^{*}\right)$ is varied through its domain to determine the $\bar{z}$ dependency of each parameter. The $C_{j}$ 's are determined by best fit to the measured data. The models are chosen as follows:

(a) $J_{ \pm}$is modeled as an exponential decay, i.e., $J_{ \pm}(\bar{z})=$ $C_{1} \exp \left(-C_{2} \bar{z}\right)$. The current is assumed to decay exponentially due to the magnetic diffusion. The extrapolation of $\bar{J}_{y}(\bar{z})$ to small $\bar{z}$ is consistent with the expected value, i.e., $J_{y \text {,expected }}=1 \mathrm{kA} /\left(\pi(0.5 \mathrm{~cm})^{2}\right) \sim$ $40 \mathrm{MAm}^{-2} \rightarrow \bar{J}_{y \text {,expected }} \sim 8 \times 10^{3}$.

(b) $\rho_{i}$ and $t_{i}$ for $i=0,1,2,3$ are fitted as second degree polynomials with respect to $\bar{z}$, for example, $t_{0}(\bar{z})=$ $C_{3} \bar{z}^{2}+C_{4} \bar{z}+C_{5}$. The Langmuir probe data covers most of the range of $\bar{z}$. Therefore, we choose the simplest smooth curve function to fit these parameters. $t_{ \pm}$lie well with the best fit of $t_{1}$ and $t_{3}$ within their domain, so we set $t_{-}(\bar{z})=t_{1}(\bar{z})$ and $t_{+}(\bar{z})=t_{3}(\bar{z})$.

(c) $\tau_{ \pm}$and $\lambda$ are chosen to be constants, i.e., $\tau_{ \pm}(\bar{z})=$ 0.01 and $\lambda(\bar{z})=0.1$. From observations, the widths do not change with $\bar{z}$ as much as the other parameters. Thus, for simplicity, the widths are assumed to be constant for all $\bar{z}$.

5. Figures 5(b) and (d) are obtained by plotting contours in the spacetime domain using the constructed functions:

$$
\begin{gathered}
\bar{J}_{y}(\bar{z}, \bar{t})=\bar{J}_{y, \text { con }}\left(\bar{t} ; J_{ \pm}(\bar{z}), t_{ \pm}(\bar{z}), \tau_{ \pm}(\bar{z})\right), \\
\bar{\rho}(\bar{z}, \bar{t})=\bar{\rho}_{\text {con }}\left(\bar{t} ; \rho_{i}(\bar{z}), t_{i}(\bar{z}), \lambda(\bar{z})\right) .
\end{gathered}
$$

\section{ORCID iDs}

Pakorn Wongwaitayakornkul (1) https://orcid.org/0000-00017455-8582

Magnus A. Haw (1) https://orcid.org/0000-0001-5739-5160

Hui Li (i) https://orcid.org/0000-0003-3556-6568

Paul M. Bellan (iD https://orcid.org/0000-0002-0886-8782

\section{References}

Attrill, G. D. R., Harra, L. K., van Driel-Gesztelyi, L., \& Démoulin, P. 2007, ApJL, 656, L101

Biesecker, D., Myers, D., Thompson, B., Hammer, D., \& Vourlidas, A. 2002, ApJ, 569, 1009
Bourdin, P.-A. 2017, ApJL, 850, L29

Carley, E. P., Long, D. M., Byrne, J. P., et al. 2013, NatPh, 9, 811

Chen, J., Marqu, C., Vourlidas, A., Krall, J., \& Schuck, P. W. 2006, ApJ, 649, 452

Chen, P., Wu, S., Shibata, K., \& Fang, C. 2002, ApJL, 572, L99

Chen, P., \& Wu, Y. 2011, ApJL, 732, L20

Chen, P. F., \& Fang, C. 2011, in "EIT Waves" and Coronal Mass Ejections, First Asia-Pacific Solar Physics Meeting ASI Conf. Ser. 2, ed. A. R. Choudhuri \& D. Banerjee (Hyderabad: ASI), 229

Cheng, X., Zhang, J., Olmedo, O., et al. 2012, ApJL, 745, L5

Cohen, O., Attrill, G. D. R., IV, \& Wills-Davey, M. J, W. B. M. 2009, ApJ, 705,587

Delannée, C., Török, T., Aulanier, G., \& Hochedez, J.-F. 2008, SoPh, 247, 123

Downs, C., Roussev, I. I., van der Holst, B., et al. 2011, ApJ, 728, 2

Fitzpatrick, R. 2014, Plasma Physics: An Introduction (Boca Raton, FL: CRC Press)

Gary, G. A. 2001, SoPh, 203, 71

Gopalswamy, N., \& Thompson, B. 2000, JASTP, 62, 1457

Greifinger, C., \& Cole, J. D. 1961, PhFl, 4, 527

Ha, B. N., \& Bellan, P. M. 2016, GeoRL, 43, 9390

Harra, L. K., \& Sterling, A. C. 2003, ApJ, 587, 429

Haw, M. A., Wongwaitayakornkul, P., Li, H., \& Bellan, P. M. 2018, ApJL, 862, L15

Hoffman, A. L. 1967, PhD thesis, Caltech http://resolver.caltech.edu/ CaltechETD:etd-12292005-135853

Klassen, A., Aurass, H., Mann, G., \& Thompson, B. 2000, A\&AS, 141, 357

Kozarev, K. A., Korreck, K. E., Lobzin, V. V., Weber, M. A., \& Schwadron, N. A. 2011, ApJL, 733, L25

Landau, L., \& Lifshitz, E. 1959, Fluid Mechanics: Course of Theoretical Physics. Vol. 6 (Oxford: Pergamon)

Li, S., \& Li, H. 2003, Los Alamos National Lab, Technical Report, LA-UR03-8926

Liu, W., Nitta, N. V., Schrijver, C. J., Title, A. M., \& Tarbell, T. D. 2010, ApJL, 723, L53

Long, D. M., Baker, D., Williams, D. R., et al. 2015, ApJ, 799, 224

Long, D. M., Bloomfield, D. S., Chen, P. F., et al. 2017, SoPh, 292, 7

Long, D. M., Gallagher, P. T., McAteer, R. J., \& Bloomfield, D. S. 2008, ApJL, 680, L81

Lynch, B. J., Antiochos, S. K., MacNeice, P. J., Zurbuchen, T. H., \& Fisk, L. A. 2004, ApJ, 617, 589

Ma, S., Raymond, J. C., Golub, L., et al. 2011, ApJ, 738, 160

Mann, G. 1995, JPIPh, 53, 109

Muhr, N., Veronig, A. M., Kienreich, I. W., et al. 2011, ApJ, 739, 89

Muhr, N., Veronig, A. M., Kienreich, I. W., et al. 2014, SoPh, 289, 4563

Ofman, L., \& Thompson, B. 2002, ApJ, 574, 440

Olmedo, O., Vourlidas, A., Zhang, J., \& Cheng, X. 2012, ApJ, 756, 143

Patsourakos, S., \& Vourlidas, A. 2009, ApJL, 700, L182

Ryutov, D. D., Drake, R. P., \& Remington, B. A. 2000, ApJS, 127, 465

Schrijver, C. J., Aulanier, G., Title, A. M., Pariat, E., \& Delanne, C. 2011, ApJ, 738,167

Simpson, J. C., Lane, J. E., Immer, C. D., \& Youngquist, R. C. 2001, NASA Technical Documents, https://ntrs.nasa.gov/archive/nasa/casi.ntrs.nasa. gov/20010038494.pdf

Stenson, E. V., \& Bellan, P. M. 2012, PhRvL, 109, 075001

Terradas, J., \& Ofman, L. 2004, ApJ, 610, 523

Thompson, B., \& Myers, D. 2009, ApJS, 183, 225

Thompson, B., Plunkett, S., Gurman, J., et al. 1998, GeoRL, 25, 2465

Tokman, M., \& Bellan, P. 2002, ApJ, 567, 1202

Vanninathan, K., Veronig, A. M., Dissauer, K., et al. 2015, ApJ, 812, 173

Veronig, A. M., Temmer, M., \& Vršnak, B. 2008, ApJL, 681, L113

Vršnak, B., \& Cliver, E. W. 2008, SoPh, 253, 215

Vršnak, B., \& Lulić, S. 2000, SoPh, 196, 157

Warmuth, A., Mann, G., \& Aurass, H. 2005, ApJL, 626, L121

Warmuth, A., Vršnak, B., Magdalenić, J., Hanslmeier, A., \& Otruba, W. 2004, A\&A, 418, 1117

Wills-Davey, M., \& Thompson, B. 1999, SoPh, 190, 467

Wills-Davey, M. J., \& Attrill, G. D. R. 2009, SSRv, 149, 325

Wills-Davey, M. J., DeForest, C. E., \& Stenflo, J. O. 2007, ApJ, 664, 556

Wongwaitayakornkul, P., Haw, M. A., Li, H., Li, S., \& Bellan, P. M. 2017, ApJ, 848, 89

Zhai, X., Li, H., Bellan, P. M., \& Li, S. 2014, ApJ, 791, 40

Zhukov, A. N. 2011, JASTP, 73, 1096

Žic, T., Vršnak, B., Temmer, M., \& Jacobs, C. 2008, SoPh, 253, 237 